\title{
Methodische Probleme \\ bei der ERstellung von Energiemodellen
}

\author{
Vorsitz: \\ A. Voss, KFA Jülich $\mathrm{GmbH}, \mathrm{STE}$
}

Die Erstellung von Energiemodellen als einem Anwendungsgebiet der noch recht jungen Wissenschaft der Systemforschung stöBt heute noch auf eine Reihe methodischer Probleme, die sich grob in zwei Kategorien einteilen lassen. Einmal in die Probleme der Anwendung mathematischer Algorithmen und Verfahren in den Modellen selbst und zum anderen in die Probleme der Beschaffung und Handhabung groBer Datenmengen. Die Diskussion im Arbeitskreis 3 orientierte sich an dieser Zweiteilung, wobei die Datenprobleme aus zeitgründen nur kurz angerissen werden konnten. Im folgenden sollen die Schwerpunkte der Diskussion und die gemeinsam erarbeiteten Ergebnisse erläutert werden.

Neue Techniken und Methoden und ihre Anwendung bei EnergiemodeIIen

Die Begriffe Techniken und Methoden sind in diesem Zusammenhang sehr weit $z u$ verstehen und umfassen sowohl die mathematischen Verfahren und Algorithmen, die dem Modell zugrunde liegen, als auch die Art und Weise der Wechselwirkung des Modells mit seinem Benutzer und schlieBlich auch das, was man mit "Ergebnisumsetzungstechnik" beschreiben könnte.

Ergebnisumsetzungstechnik meint in diesem zusammenhang alle Verfahren und Möglichkeiten, die Ergebnisse der Modellrechnungen dem Entscheidungsträger so näher zu bringen, daß sie als Entscheidungshilfe genutzt werden. Prinzipiell lassen sich drei Strategien unterscheiden, die jeweils eine gewisse Rückwirkung auf den Aufbau des Modelis selbst haben.

1. Man entwickelt einfache Modelle, deren Struktur im Detail für den Entscheidungsträger durchschaubar ist und die der Entscheidungsträger selbst benutzt. Die Aussagefähigkeit dieser Art von Modellen ist beschränkt, und es besteht die Gefahr, daB der Entscheidungsträger sich der Einschränkungen nicht bewust ist. 
2. Entwicklung komplexer Modelle, die den Problemstellungen adäquat sind und vom Entscheidungsträger benutzt werden sollen. Die Wahrscheinlichkeit, daß der Benutzer sich des Modells nicht bedient, weil er die zusammenhänge und den Aufbau dieses komplexen Modells nicht versteht, ist sehr groB.

3. Die dritte Strategie besteht in der Entwicklung problemadäquater Modelle, die nicht vom Entscheidungsträger selbst benutzt werden. Hier kommt der Kommunikation zwischen dem, der mit dem Modell rechnet, und dem Entscheidenden eine wichtige Rolle bei der Schaffung des für die Entscheidung notwendigen Vertrauens in die Modellergebnisse zu.

Der Bedeutung der Umsetzung der Modellergebnisse wird oft von den Modellbauern nicht die notwendige Beachtung geschenkt. Gute Ergebnisse, die in der Schublade verschwinden, nutzen aber nichts.

Diskutiert wurde fernerdie Möglichkeit derEntwicklung adaptiver Modelle sowie die Anwendung der Netztechniken (Petri-Netze) im Rahmen von Energiemodellen.

Versuch einer Wertung und Einordnung der spezifischen Aussagemöglichkeiten und Anwendungsgebiete verschiedener methodischer Ansätze

Die heute im Rahmen von Energiemodellen verwendeten Ansätze lassen sich einteilen in

Optimierungsansätze,

kybernetische Simulationsansätze und ökonometrische Ansätze.

Optimierungsmodelle minimieren oder maximieren eine zielfunktion unter Beachtung einer Reihe von restriktiven Nebenbedingungen. Unter kybernetischer Simulation sind hier Modelle einzuordnen, die die Entwicklung von Zeitreihen der Modellgrößen endogen erklären, z.B. durch die Lösung von Differentialgleichungen nach Vorgabe eines Anfangszustandes. Ckonometrische Ansätze ermitteln Zusammenhänge aus der Vergangenheit und schreiben sie für die zukunft fort.

\begin{tabular}{|l|c|c|c|}
\cline { 2 - 4 } \multicolumn{1}{c|}{} & Dynamik & Allokation & $\begin{array}{c}\text { stat. Auswir- } \\
\text { kungsanalyse }\end{array}$ \\
\hline Pptimierung & - & 1 & 2 \\
kyb. Simulation & 1 & 2 & - \\
bkonometrie & - & 2 & 1 \\
\hline
\end{tabular}

1 = gut

2 = weniger gut 
Dieses Schema versucht nun ganz grob, diese drei Ansätze nach ihren methodisch-spezifischen Aussagemöglichkeiten zu klassifizieren. Es wird dabei unterschieden nach der jeweiligen Eignung zur Beschreibung der Dynamik einer Entwicklung, zur Beschreibung von Zuordnungsproblemen und zur Ermittlung der Auswirkungen von Veränderungen bei einer statischen Betrachtungsweise.

Es bleibt festzustełlen, daß die ziele der Modellentwicklung bestimmend sind für die Auswahl der $\mathrm{zu}$ verwendenden Methode. Eine Kombination der verschiedenen methodischen Ansätze wird dabei in der Regel sinnvoll sein, um die jeweiligen spezifischen Vorteile zu nutzen.

\section{Probleme der Regionalisierung}

Die Erfassung ortsabhängiger Effekte in Energiemodellen wie z.B. die Energieverteilung, die Standortfragen von Kraftwerken oder die Ortsabhängigkeit des Energieverbrauchs, bereitet heute noch eine Reihe von Schwierigkeiten. Da diese Fragen unter Umständen eine wichtige Rolle bei der Beurteilung zukünftiger Energieversorgungssysteme spielen, ist es notwendig, sie methodisch adäquat in Energiemodellen $\mathrm{zu}$ behandeln. Drei Verfahrensweisen scheinen möglich:

1. Die zu betrachtende Region wird in Teilregionen aufgeteilt, deren räumliche Abgrenzung geeignet ist, die ortsabhängigen Einflüsse zu erfassen. Die Modelle der Teilregionen müssen dann verknüpft werden, um die Wechselwirkungen zwischen den Teilregionen (z.B. Ausbreitung der Luftbelastung) zu erfassen.

2. Für die Probleme der Regionalisierung werden eigene Modelle entwickelt, z.B. Transport- und Verteilungsmodelle. Sie werden mit dem Globalmodell in einem iterativen Proze $\beta$ betrieben, d.h. die Ergebnisse des Globalmodells werden in bezug auf spezifische Teilaspekte in den Modellen für die Einzelfragen überprüft.

3. Im Rahmen des Globalmodells werden die ortsabhängigen Effekte über Verteilungsfunktionen, z.B. für die Energieverbrauchsdichte und über spezielle strategierechnungen, z.B. für verschiedene Standortkonzepte und die damit verbundenen Transportprobleme erfaßt.

\section{Datenhandhabung}

Die Probleme der Datenbeschaffung und der Handhabung großer Datenmengen konnten aus Zeitgründen nur andiskutiert werden. Deshalb ist es auch nur möglich, die Probleme kurz aufzulisten und ihre Bedeutung im Rahmen einer Modellanalyse noch einmal zu unterstreichen:

- Nichtverfügbarkeit wichtiger Daten, z.B. über den Nutzenergiebedarf.

- Probleme der Verarbeitung großer Datenmengen.

- Probleme der Ergebnisauswertung begründet in einer zu großen Menge erzeugter Daten und. Informationen. 